\title{
Relaciones entre el conocimiento conceptual y el procedimental en el aprendizaje de las fracciones
}

\section{Relationships between conceptual and procedural knowledge in fractions learning}

ISSN 1510-2432 - ISSN 1688-9304 (en línea) - DOI: http://dx.doi.org/10.18861/cied.2016.7.1.2573

Florencia Stelzer

Doctora en Psicología, Facultad de Psicología, Universidad Nacional de Mar del Plata. Becaria Post doctoral, Concejo Nacional de Investigaciones Científicas y Técnicas (CONICET) Argentina.

María Laura Andrés

Doctora en Psicología, Facultad de Psicología,Universidad Nacional de Mar del Plata. Becaria Post Doctoral, Concejo Nacional de Investigaciones Científicas y Técnicas (CONICET), Argentina.

Lorena Canet-Juric

Doctora en Psicología, Facultad de Psicología, Universidad Nacional de San Luis. Investigadora Asistente, Concejo Nacional de Investigaciones Científicas y Técnicas (CONICET) Argentina.

Isabel Introzzi

Doctora en Psicología, Facultad de Psicología, Universidad Nacional de San Luis. Investigadora Adjunta,Concejo Nacional de Investigaciones Científicas y Técnicas (CONICET) Argentina, Centro de Investigación en Procesos Básicos, Metodología y Educación (CIMEPB).

Sebastián Urquijo

Doctor en Educación, área de Psicología Educacional, Faculdade de Educação, Universidade Estadual de Campinas (UNICAMP). Investigador Independiente, Concejo Nacional de Investigaciones Científicas y Técnicas (CONICET) Argentina. Profesor adjunto en las cátedras de Psicología Cognitiva y de Teorías del Aprendizaje, Facultad de Psicología, Universidad Nacional de Mar del Plata.

Fecha de recibido: 07/03/2016

Fecha de aceptado: 05/04/2016

\section{Resumen}

El objetivo de este trabajo fue analizar las relaciones entre el conocimiento conceptual y el procedimental de las fracciones durante su aprendizaje. Para esto se efectuó una búsqueda bibliográfica en las bases de datos ERIC, Psyclnfo, Scielo y Redalyc, con los siguientes términos en español y sus equivalentes en inglés: fracciones (fractions), conocimiento conceptual (conceptual knowledge), conocimiento procedimental (procedural knowledge) y niños (children), combinados de diferente forma con el operador booleano AND (Y). Los resultados de esta búsqueda permitieron hallar quince artículos empíricos que pueden clasificarse en cuatro grupos de investigaciones: las que indican una relación bidireccional, las que sugieren una relación unidireccional, las que muestran cierta independencia o restricciones en su vinculación y, por último, las que señalan que las relaciones varían según los sujetos. Esta falta de acuerdo podría explicarse por ciertas diferencias metodológicas de los estudios, por ejemplo, diferencias en el grado de consolidación del conocimiento de las fracciones de los participantes, el sentido conceptual o la habilidad procedimental estudiada, el tipo de enseñanza matemática recibida, etc. Por ello se sugiere que, para profundizar la comprensión de las relaciones entre el conocimiento conceptual y procedimental de las fracciones, estos aspectos metodológicos deben ser controlados.

Palabras clave: aprendizaje matemático, fracciones, conocimiento conceptual, conocimiento procedimental, niños 


\begin{abstract}
The aim of this study was to analyze the relationship between conceptual knowledge and procedural knowledge of fractions during learning. For this propose, a literature search was conducted in the databases of ERIC, PsycINFO, Scielo and Redalyc, using the following terms in English and their equivalent word in Spanish: Fractions (fracciones), conceptual knowledge (conocimiento conceptual), procedural knowledge (conocimiento procedimental) and children (niños). The terms were combined in different ways using the Boolean operator AND. The results of this search produced fifteen empirical articles that can be classified into four research groups: those that indicate a bidirectional relationship, those that suggest unidirectional relationship, those that showed some independence or restrictions on their relationship and those stating that relationships vary according to subjects. This lack of agreement could be explained by some methodological differences among studies, for example, differences in the consolidation degree of fraction knowledge of the participants, the conceptual sense or procedural ability studied, the type of mathematical education received, etc. Therefore, so as to deepen the understanding of the relationship between conceptual and procedural knowledge of fractions, we suggest that such methodological aspects should be controlled.
\end{abstract}

Key words: mathematical learning, fractions, conceptual knowledge, procedural knowledge, children

\title{
Relaciones entre el conocimiento conceptual y el procedimental en el aprendizaje de las fracciones
}

El conocimiento de las matemáticas contribuye al bienestar individual y al desarrollo de las sociedades, predice las oportunidades laborales, el éxito profesional y el nivel de ingresos (Ancker \& Kaufman, 2007; Grinyer, 2005; Paglin \& Rufolo, 1990; Parsons \& Bynner, 2005; Rivera-Batiz, 1992; Rose \& Betts, 2004), así como posibilita el desarrollo científicotecnológico. Obviamente, la adecuada capacitación de los ciudadanos en esta área impulsa el crecimiento económico y cultural (Clark, 1988; National Mathematics Advisory Panel (NMAP), 2008; Parsons \& Bynner, 2005) por lo cual, la comprensión de las variables que explican el mismo presenta una particular importancia.

Dentro del campo de la cognición matemática existe cierto consenso en diferenciar dos grandes formas o tipos de conocimientos: el conceptual y el procedimental (Rittle-Johnson \& Schneider, 2014). Por una parte, el conocimiento conceptual considera los principios abstractos que rigen un dominio y sus interrelaciones. Por otra parte, el conocimiento procedimental refiere a la capacidad de ejecución de los diferentes pasos o algoritmos requeridos para la resolución de un problema (Bisanz \& LeFevre, 1992; Rittle-Johnson, Siegler \& Alibali, 2001). Aunque actualmente se admite que durante el aprendizaje existe una asociación entre ambos tipos de conocimiento, se observan discrepancias respecto de la forma en la que se produce (Byrnes \& Wasik, 1991; Greeno, Riley \& Gelman, 1984; Hiebert, 2013; Hiebert \& Wearne, 1986; Rittle-Johnson, Schneider \& Star, 2015; Rittle-Johnson \& Schneider, 2014). La comprensión de esta relación impacta en la política educativa dado que determina cómo deben ser impartidos los contenidos de las matemáticas (Hiebert, 2013).

En la literatura pueden distinguirse tres grandes perspectivas sobre las relaciones de estos conocimientos entre sí. Por un lado, ciertos investigadores consideran que los niños adquieren primero conocimientos conceptuales y, a partir de los mismos y de la experiencia en la resolución de problemas construyen y perfeccionan el conocimiento procedimental (e.g., Geary, 1994; Gelman \& Williams, 1998; Halford, 2014). Por otro lado, y en un sentido contrario, otros autores sugieren que los niños incorporan primero los procedimientos sin una clara comprensión de los principios conceptuales subyacentes y luego, a través de 
la abstracción, serían capaces de comprender progresivamente sus bases conceptuales (e.g., Fuson, 1998; Karmiloff-Smith, 1992; Siegler \& Stern, 1998). Por último, algunos autores sugieren que ambas formas de conocimiento se adquieren simultáneamente y de forma interactiva, de modo tal que el incremento en un tipo de conocimiento repercute positivamente en el otro y viceversa (e.g., Baroody, 2003; Rittle-Johnson \& Siegler, 1998; Rittle-Johnson et al., 2001).

Estudios de revisión recientes sobre la asociación entre ambas formas de conocimiento (Rittle-Johnson \& Schneider, 2014) sugieren que los hallazgos contradictorios entre las investigaciones podrían deberse a sus diferencias en el contenido curricular considerado (e.g., números naturales, números racionales, operaciones algebraicas, entre otros) y/o en el nivel de aprendizaje del mismo. De este modo, para una mejor comprensión de sus relaciones sería necesario considerar estos aspectos.

Dentro de los contenidos curriculares de la educación primaria, los números fraccionarios -y con ellos las operaciones- son de particular importancia. Por una parte, teorías recientes del desarrollo numérico (Siegler, Thompson \& Schneider, 2011) indican que el aprendizaje de las fracciones propicia una comprensión más profunda y madura del sistema numérico. Esto se debería a que la enseñanza de las fracciones introduce una serie de propiedades no aplicables a los números naturales. Por ejemplo, a diferencia de los números naturales, la numerosidad de una fracción puede ser representada a través de otros números fraccionarios, los números fraccionarios disminuyen con la multiplicación y se incrementan con la división, y así. Tal ampliación del conocimiento numérico impulsaría a los niños a reformular y a madurar su conocimiento conceptual de los números.

Por otra parte, el conocimiento de las fracciones durante la educación primaria predice la adquisición del conocimiento del álgebra y del aprendizaje general de la matemática durante la secundaria (Siegler et al., 2012). Esta relación se sostiene incluso al controlar diferentes variables que han sido señaladas como predictores significativos del aprendizaje de la matemática, tales como la inteligencia, la memoria de trabajo, la capacidad de lectura, el nivel de ingreso y de educación familiar (Siegler et al., 2012). El conocimiento del álgebra conforma una base fundamental en las carreras tecnológicas, de ingeniería y matemática de formación superior, por lo cual los individuos que tienen dificultades en este dominio experimentan mayores dificultades y mayor deserción académica en tales carreras (NMAP, 2008; Sadler \& Tai, 2001).

Lo anteriormente expuesto muestra la relevancia del aprendizaje de las fracciones. Sin embargo, muchos niños experimentan dificultades en este proceso más allá de recibir una adecuada enseñanza (Yoshida \& Sawano, 2002; Hoffer, Venkataraman, Hedberg \& Shagle, 2007; Vamvakossi \& Vosniadou, 2004; 2010). Estas dificultades se observan tanto en la adquisición del conocimiento conceptual como en la incorporación de los procedimientos necesarios para efectuar operaciones con números fraccionarios.

Ciertos autores (Behr, Harel, Post \& Lesh, 1992; Kieren, 1976; 1993) han propuesto que el conocimiento conceptual de las fracciones involucra la comprensión de los diferentes significados o sentidos de las mismas. Es decir, diferentes representaciones o nociones conceptuales subyacen a este conocimiento. Se ha considerado a las fracciones como: (a) una relación parte-entero (asociación entre un entero y un número de partes iguales en las cuales se divide), (b) un cociente (como el resultado de una operación de reparto), (c) un operador (como una función operatoria aplicada a otra magnitud; e.g., 3/4 a, siendo a=1/2 , la operatoria es $1 \times 3 / 2 \times 4$ ), (d) una razón (como un índice comparativo entre dos cantidades), 
y (e) una forma de medición de la magnitud (como un modo de representación de las magnitudes) (Kieren, 1976; 1993; Behr et al., 1992; Charalambous \& Pitta-Pantazi, 2005; Vizcarra \& Sallán, 2005). Estos diferentes significados de las fracciones estarían incluidos en las distintas actividades curriculares propuestas durante la instrucción matemática (La Tabla 1 contiene ejemplos de actividades que evalúan los diferentes sentidos).

Asimismo, el conocimiento procedimental de las fracciones implica el conocimiento de los pasos necesarios para la resolución de un problema que involucre tal formato numérico (e.g., conocimiento de los pasos para efectuar operaciones de adición de fracciones) (Bisanz \& LeFevre, 1992; Rittle-Johnson, Siegler \& Alibali, 2001).

\section{Tabla 1.Principales tareas utilizadas para la medición de los diferentes sentidos de las fracciones}

\begin{tabular}{|c|c|c|c|c|}
\hline $\begin{array}{l}\text { Sentido } \\
\text { evaluado }\end{array}$ & $\begin{array}{c}\text { Nombre de la } \\
\text { tarea* }\end{array}$ & $\begin{array}{l}\text { Descripción } \\
\text { general }\end{array}$ & Ejemplo & $\begin{array}{c}\text { Estudios que la } \\
\text { utilizaron }\end{array}$ \\
\hline \multirow[t]{3}{*}{$\begin{array}{l}\text { Relación parte- } \\
\text { entero }\end{array}$} & $\begin{array}{l}\text { Gráfico - } \\
\text { símbolo }\end{array}$ & $\begin{array}{l}\text { Indicar el } \\
\text { número } \\
\text { fraccionario } \\
\text { que } \\
\text { representada } \\
\text { un gráfico }\end{array}$ & $\begin{array}{c}\square \square \\
\text { Indique el número } \\
\text { fraccionario que } \\
\text { representa este gráfico }\end{array}$ & $\begin{array}{l}\text { Byrnes \& Wasik (1991); } \\
\text { Charalambous et al. } \\
\text { (2005); Hetch \& Vagi } \\
\text { (2010; 2012); Osana \& } \\
\text { Pitsolantis (2013) }\end{array}$ \\
\hline & $\begin{array}{l}\text { Símbolo - } \\
\text { gráfico }\end{array}$ & $\begin{array}{l}\text { Representar } \\
\text { gráficamente } \\
\text { una fracción } \\
\text { presentada } \\
\text { en números } \\
\text { arábigos }\end{array}$ & $\begin{array}{l}\text { Represente en el } \\
\text { siguiente cuadrado la } \\
\text { fracción 3/4 } \\
\square\end{array}$ & $\begin{array}{c}\text { Fuchs et al. (2013; 2014); } \\
\text { Hallet et al. (2010; 2012); } \\
\text { Hecht \& Vagi (2010; } \\
\text { 2012); Osana \& Pitsolantis } \\
\text { (2013); }\end{array}$ \\
\hline & $\begin{array}{l}\text { Formas } \\
\text { simples }\end{array}$ & $\begin{array}{l}\text { Identificar } \\
\text { fracciones } \\
\text { equivalentes } \\
\text { representadas }\end{array}$ & $\begin{array}{l}\text { Indique cuáles de los } \\
\text { siguientes gráficos } \\
\text { representan la misma } \\
\text { cantidad }\end{array}$ & Byrnes \& Wasik (1991) \\
\hline \multicolumn{5}{|c|}{ * Las tareas pueden haber sido designadas con diferentes términos en distintos estudios } \\
\hline \multirow[t]{4}{*}{$\begin{array}{l}\text { Medición de la } \\
\text { magnitud }\end{array}$} & $\begin{array}{l}\text { Relaciones a } \\
\text { valores fijos }\end{array}$ & $\begin{array}{l}\text { Seleccionar la } \\
\text { fracción más } \\
\text { próxima, mayor } \\
\text { o menor a "x" } \\
\text { valor }\end{array}$ & $\begin{array}{l}\text { Indique entre las } \\
\text { siguientes fracciones: } \\
3 / 4 ; 4 / 8 ; 1 / 3 \text {, cuál es la } \\
\text { más cercana a } 1 / 2\end{array}$ & Rittle-Jhonson (2001) \\
\hline & $\begin{array}{l}\text { Cantidad } \\
\text { continua }\end{array}$ & $\begin{array}{c}\text { Escribir el } \\
\text { número que } \\
\text { se ubica entre } \\
\text { las fracciones } \\
\text { decimales "x" } \\
\text { e "y" }\end{array}$ & $\begin{array}{c}\text { Escriba un numero } \\
\text { fraccionario que se situé } \\
\text { entre } 1 / 4 \text { y } 3 / 4\end{array}$ & $\begin{array}{l}\text { Charalambous et al. } \\
\text { (2005); } \\
\text { Rittle-Johnson (2001) }\end{array}$ \\
\hline & $\begin{array}{c}\text { Valores } \\
\text { equivalentes }\end{array}$ & $\begin{array}{l}\text { Indicar los } \\
\text { valores } \\
\text { equivalentes a } \\
\text { "x" fracción }\end{array}$ & $\begin{array}{l}\text { Indique una fracción } \\
\text { equivalente a } 4 / 8\end{array}$ & $\begin{array}{l}\text { Hallet et al. (2010; 2012); } \\
\text { Rittle-Johnson (2001) }\end{array}$ \\
\hline & $\begin{array}{l}\text { Estimación del } \\
\text { resultado de } \\
\text { operaciones } \\
\text { aritméticas }\end{array}$ & $\begin{array}{l}\text { Indicar el } \\
\text { resultado más } \\
\text { cercano para } \\
\text { una operación } \\
\text { aritmética con } \\
\text { fracciones }\end{array}$ & $\begin{array}{c}99 / 100+99 / 100=1 ; 10 \\
100 \text { ó } 1,000 ?\end{array}$ & $\begin{array}{l}\text { Hallet et al. (2010; 2012); } \\
\text { Hetch \& Vagi (2010; 2012); } \\
\text { Rittle-Johnson (2001) }\end{array}$ \\
\hline
\end{tabular}




\begin{tabular}{|c|c|c|c|c|}
\hline & $\begin{array}{l}\text { Ubicación } \\
\text { en la línea } \\
\text { numérica }\end{array}$ & $\begin{array}{l}\text { Ubicar } \\
\text { números } \\
\text { fraccionarios } \\
\text { en la línea } \\
\text { numérica }\end{array}$ & $\begin{array}{c}-2 \ldots-1 \ldots 0 \_-+1 \_^{-+2} \\
\text { Ubique los números } 1 / 2 ; \\
8 / 4 ;-3 / 4\end{array}$ & $\begin{array}{l}\text { Charalambous et al. } \\
\text { (2007); Fuchs et al. (2013; } \\
\text { 2014); Hallet et al., (2010) }\end{array}$ \\
\hline & $\begin{array}{l}\text { Comparación } \\
\text { numérica }\end{array}$ & $\begin{array}{l}\text { Indicar cuál de } \\
\text { dos números } \\
\text { es mayor }\end{array}$ & $\begin{array}{c}\text { Señale el número de } \\
\text { mayor valor } \\
\\
\text { ¿1/2 vs. } 1 ? \\
i 4 / 2 \text { vs. } 2 ? \\
i 2 / 4 \text { vs. } 2 / 16 ?\end{array}$ & $\begin{array}{l}\text { Fuchs et al. (2013); Hallet } \\
\text { et al. (2010; 2012); Hetch } \\
\text { \& Vagi (2010; 2012); } \\
\text { Rittle-Jhonson (2001) }\end{array}$ \\
\hline \multirow[t]{2}{*}{$\begin{array}{l}\text { Conocimiento } \\
\text { conceptual de } \\
\text { adición }\end{array}$} & $\begin{array}{l}\text { Computación } \\
\text { con gráficos }\end{array}$ & $\begin{array}{l}\text { Computar } \\
\text { operaciones } \\
\text { aritméticas } \\
\text { con fracciones } \\
\text { presentadas } \\
\text { gráficamente }\end{array}$ & $+\square$ & Hetch \& Vagi $(2010 ; 2012)$ \\
\hline & $\begin{array}{l}\text { Representar } \\
\text { cómputos } \\
\text { aritméticos } \\
\text { con gráficos }\end{array}$ & $\begin{array}{l}\text { Representar } \\
\text { cómputos } \\
\text { aritméticos con } \\
\text { gráficos }\end{array}$ & $\begin{array}{c}\text { Represente } \\
\text { gráficamente la } \\
\text { operación } 2 / 5+3 / 5\end{array}$ & Osana \& Pitsolantis (2013) \\
\hline $\begin{array}{l}\text { Conocimiento } \\
\text { conceptual de } \\
\text { sustracción }\end{array}$ & $\begin{array}{l}\text { Argumentación } \\
\text { explicativa }\end{array}$ & $\begin{array}{l}\text { Argumentar el } \\
\text { motivo por el } \\
\text { que se ejecutan } \\
\text { determinados } \\
\text { pasos y/o } \\
\text { se obtienen } \\
\text { ciertos } \\
\text { resultados }\end{array}$ & $\begin{array}{c}538-259=(500-200)+ \\
(30-0)+(8-9) \text { ¿Por qué? }\end{array}$ & Peled \& Segalis (2005) \\
\hline Razón & $\begin{array}{l}\text { Ítems que } \\
\text { miden el } \\
\text { sentido de } \\
\text { razón }\end{array}$ & $\begin{array}{l}\text { Problemas } \\
\text { verbales que } \\
\text { requieren } \\
\text { establecer } \\
\text { comparaciones } \\
\text { entre dos } \\
\text { números } \\
\text { fraccionarios }\end{array}$ & $\begin{array}{c}\text { Ocho niñas se reparten } \\
3 \text { pizzas mientras que } \\
3 \text { niños se reparten } \\
1 \text { pizza. ¿Quiénes } \\
\text { comerán más pizza, los } \\
\text { niños o las niñas? }\end{array}$ & $\begin{array}{c}\text { Byrnes \& Wasik (1991); } \\
\text { Charalambous et al. } \\
\text { (2007); Hallet et al. (2012) }\end{array}$ \\
\hline Operación & $\begin{array}{l}\text { Ítems que } \\
\text { miden el } \\
\text { sentido de } \\
\text { operación }\end{array}$ & $\begin{array}{l}\text { Problemas } \\
\text { verbales que } \\
\text { requieren } \\
\text { operar con } \\
\text { fracciones }\end{array}$ & $\begin{array}{l}\text { Sin efectuar el cálculo, } \\
\text { señale si el siguiente } \\
\text { enunciado es correcto: } \\
\text { "Si se divide un número } \\
\text { por } 4 \text { y luego se } \\
\text { multiplica el mismo } \\
\text { por } 3 \text { se obtendría el } \\
\text { mismo resultado que } \\
\text { si multiplicáramos tal } \\
\text { número por 3/4". }\end{array}$ & $\begin{array}{l}\text { Charalambous et al. } \\
\text { (2007) }\end{array}$ \\
\hline Cociente & $\begin{array}{l}\text { Ítems que } \\
\text { miden el } \\
\text { sentido de } \\
\text { cociente }\end{array}$ & $\begin{array}{c}\text { Problemas } \\
\text { verbales que } \\
\text { implican } \\
\text { operaciones de } \\
\text { división }\end{array}$ & $\begin{array}{c}\text { Decida si la siguiente } \\
\text { afirmación es correcta } \\
\text { "2/3 es igual al resultado } \\
\text { de la división de } 2 \text { sobre } \\
\text { 3". }\end{array}$ & $\begin{array}{l}\text { Charalambous et } \\
\text { al. (2007); Osana \& } \\
\text { Pitsolantis (2013) }\end{array}$ \\
\hline
\end{tabular}

Considerando la importancia del adecuado aprendizaje de las fracciones y teniendo presente que su adquisición representa un desafío para muchos niños, la comprensión del aprendizaje de este conocimiento reviste un particular interés. Por ello, el presente trabajo se propone como objetivo realizar una revisión de la literatura que permita identificar las maneras en las que el conocimiento conceptual y el procedimental de las fracciones se relacionan durante su aprendizaje. Esto representará un aporte para los modelos teóricos del aprendizaje del conocimiento de las fracciones y proporcionará herramientas prácticas para la identificación y tratamiento de niños con dificultades en su aprendizaje. 


\section{Metodología}

Se realizó una búsqueda bibliográfica de artículos científicos disponibles como texto completo en las bases de datos ERIC y PsycInfo, Scielo, Redalyc, utilizando los términos en español y sus equivalentes en inglés: conocimiento conceptual (conceptual knowledge), conocimiento procedimental (procedural knowledge), fracciones (fractions) y niños (children) combinados con el operador booleano "AND" (Y). Se consideró como criterio de inclusión en esta revisión que los estudios estén publicados en revistas con referato, que sean de carácter empírico y que hayan sido realizados con niños.

\section{Resultados}

La revisión de la literatura indica que el conocimiento conceptual y el procedimental de las fracciones se encuentran vinculados entre sí. No obstante, tal como se adelantara en la introducción, el sentido de la dirección de tal relación es controvertido (Hetch \& Vagi, 2010; Rittle-Johnson et al., 2001). Los estudios hallados pueden ser clasificados en cuatro grupos: (a) los que indican que la asociación entre ambos es bidireccional o recíproca (Hetch \& Vagi, 2010; Rittle-Johnson et al., 2001), (b) los que sugieren que la asociación es unidireccional (Byrnes \& Wasik, 1991; Charalambous \& Pitta- Pantazi, 2007; Fuchs et al., 2013; 2014), (c) los que señalan cierta independencia entre ambas formas de conocimiento (Kerslake, 1986; Peck \& Jencks, 1981) o que su relación depende de otras variables (Mack, 1990; Osana \& Pitsolantis, 2013; Peled \& Segalis, 2005) y (d) los que plantean diferencias inter-individuales en el grado de dominio respectivo de ambos tipos de conocimiento (Hallett, Nunes \& Bryant, 2010; Hallett, Nunes, Bryant \& Thorpe, 2012; Hetch \& Vagi, 2012).

\section{Evidencia a favor de un vínculo bidireccional o recíproco}

La evidencia empírica a favor de un patrón de relación bidireccional entre el conocimiento conceptual y el procedimental de las fracciones proviene de dos estudios. El primero fue efectuado por Rittle-Johnson et al. (2001) con niños de 5. año. Estos autores encontraron que el conocimiento conceptual de las fracciones (sentido de medición de la magnitud) explicaba los cambios en la capacidad procedimental de ubicar fracciones en la línea numérica tras su entrenamiento. Asimismo, las mejoras en la capacidad procedimental de ubicar fracciones en la línea numérica afectaban el conocimiento conceptual de las fracciones (sentido medición de la magnitud) post-entrenamiento.

El segundo trabajo fue realizado por Hetch y Vagi (2010). Estos autores analizaron el grado en el que el desarrollo del conocimiento conceptual y procedimental de las fracciones durante el transcurso de $4 .^{\circ}$ a $5 .^{\circ}$ año era explicado por el desempeño en ambas formas de conocimiento en $4 .^{\circ}$ año. Sus resultados indicaron que el conocimiento conceptual de la operación de adición de fracciones y del sentido de estas como una forma de medición de la magnitud y una relación parte-entero predecía el aprendizaje de las habilidades procedimentales de efectuar y estimar cómputos aritméticos y resolver problemas verbales con fracciones durante el pasaje de $4 .^{\circ}$ a $5 .^{\circ}$ año. Asimismo, cuando consideraron la influencia del desempeño en dichas habilidades procedimentales en $4 .^{\circ}$ año sobre los cambios en el conocimiento conceptual entre $4 .^{\circ}$ y $5 .^{\circ}$ año hallaron que la capacidad de resolver problemas verbales explicaba los cambios en los tres aspectos conceptuales considerados, mientras que la capacidad de realizar cómputos aritméticos y estimar los resultados de operaciones con fracciones predecían el aprendizaje del conocimiento conceptual de adición de fracciones y la comprensión del sentido de medición de las mismas.

Los resultados de Hetch y Vagi (2010) sugieren, por una parte, que el conocimiento conceptual tendría un efecto más general y amplio sobre el aprendizaje de los procedimientos respecto 
del efecto del conocimiento procedimental sobre el aprendizaje de los conceptos. Por otra parte, es importante destacar que estos autores controlaron la influencia del cociente intelectual y de la capacidad de lectura en el análisis de las relaciones mencionadas.

\section{Evidencia a favor de un vínculo unidireccional}

Son tres las investigaciones halladas que aportaron evidencia a favor de un sentido de relación unidireccional entre ambas formas de conocimiento. Byrnes y Wasik (1991) indicaron que el conocimiento conceptual de las fracciones (relación parte-entero, razón y sentido de medición) precede y explica el aprendizaje de la capacidad de realizar procedimientos de adición y multiplicación con fracciones (Byrnes \& Wasik, 1991). En un sentido semejante, Charalambous \& Pitta-Pantazi (2007) sugirieron que el conocimiento conceptual predice el desempeño procedimental. Sin embargo, indicaron que la relación entre ambas formas de conocimiento se restringía a dimensiones específicas. Tales autores analizaron la relación en niños de $5 .^{\circ}$ y $6 .^{\circ}$ año entre los sentidos de las fracciones como una relación parte-entero, medición, razón, cociente y operador, y las habilidades procedimentales de identificar fracciones equivalentes y realizar cómputos aritméticos con fracciones. Sus resultados indicaron que la capacidad procedimental de identificar fracciones equivalentes era explicada por la comprensión del significado de las fracciones como razón, mientras que la capacidad procedimental de realizar operaciones aritméticas de multiplicación con fracciones era explicada por la comprensión del sentido de las fracciones como cociente y operador. Finalmente, la capacidad de realizar operaciones de adición con fracciones era explicada por la comprensión de las fracciones como una relación parte-entero. Sin embargo, las dos capacidades procedimentales evaluadas eran independientes de la comprensión de las fracciones como medición.

Estudios experimentales también mostraron restricciones en la asociación entre ambas formas de conocimiento, aunque la especificidad hallada sería parcialmente contraria a lo señalado por Charalambous \& Pitta-Pantazi (2007). Fuchs et al. (2013; 2014) informaron que en $4 .^{\circ}$ año la instrucción centrada en el sentido de las fracciones como medición producía un mayor aprendizaje de la capacidad de efectuar cómputos aritméticos con fracciones respecto de la instrucción centrada en el sentido de las fracciones como una relación parte-entero. Asimismo, el primer tipo de enseñanza generaba mayores mejoras en la comprensión conceptual de las fracciones (como medición y como relación parte-entero) respecto de la enseñanza centrada en la fracción como relación parte-entero (Fuchs et al., 2013). Asimismo, sus resultados mostraron que el incremento en la comprensión del sentido de las fracciones como medición mediaba el impacto del tipo de enseñanza recibida sobre el desempeño general en fracciones (conocimiento conceptual y procedimental). De este modo, a diferencia del trabajo de Charalambous \& Pitta-Pantazi, (2007), los hallazgos de Fuchs et al. $(2013 ;$ 2014) sugieren que la enseñanza centrada en el sentido de las fracciones como medición sería clave para el aprendizaje del conocimiento de fracciones.

\section{Evidencia a favor de una relativa independencia o restricciones en su vinculación}

Algunos autores (Kerslake, 1986; Peck \& Jencks, 1981) observaron que los niños son capaces de ejecutar procedimientos de cómputo con fracciones sin presentar una clara comprensión de sus bases conceptuales. Peck y Jencks (1981) reportaron que menos del $10 \%$ de los estudiantes de $6 .^{\circ}$ año demostraban una adecuada comprensión conceptual de las fracciones (relación parte-entero, sentido de medición). No obstante, aproximadamente el 35\% de los mismos eran capaces de utilizar adecuadamente procedimientos de cómputo aritmético con fracciones. En un sentido semejante, Kerslake (1986) indicó que un número notorio de niños eran capaces de resolver problemas de adición con fracciones pero no podían explicar las bases conceptuales del procedimiento utilizado. 
Desde otra perspectiva, ciertas investigaciones indican que la relación entre el conocimiento conceptual y procedimental depende de la intensidad con la que se acentúan las relaciones entre ambos durante la instrucción. Por ejemplo, Peled y Segalis (2005) indicaron que la enseñanza que acentúa las bases conceptuales del procedimiento de sustracción facilita un mejor aprendizaje de dicho conocimiento respecto de la enseñanza que no lo hace. Mack (1990) mostró que la enseñanza que utiliza el conocimiento intuitivo que los niños poseen de los números fraccionarios para la resolución de situaciones problemáticas simples y que conecta tal conocimiento con los procedimientos y la representación simbólica de las fracciones, posibilita un mejor aprendizaje conceptual (sentido de medición de la magnitud y relación parte-entero) y procedimental de las mismas respecto de la instrucción que no considera ni el conocimiento inicial ni su vinculación con problemáticas concretas. En un sentido semejante, Osana y Pitsolantis (2013) indicaron que la enseñanza que establece conexiones explícitas entre los aspectos conceptuales (relación parte-entero, sentido como magnitud, sentido de cociente, conocimiento conceptual de adición y sustracción) y procedimentales (computo aritmético) de las fracciones, produce un mayor aprendizaje del conocimiento conceptual y de la capacidad de establecer relaciones entre ambas formas de conocimiento respecto de la enseñanza que no acentúa las vinculaciones entre ambos. Por último, ciertos estudios sugieren que las diferencias individuales en las habilidades cognitivas moderan la relación específica entre el conocimiento conceptual y el procedimental (Fuchs et al., 2013, 2014). Las variables moderadoras permiten contrastar subgrupos de mayor influencia en la relación de una variable independiente con una variable dependiente (Baron \& Kenny, 1986).

En el caso del aprendizaje de las fracciones, algunos estudios analizaron si el aprendizaje de las fracciones resultante de diferentes tipos de instrucción se encontraba moderado por distintas habilidades cognitivas (Fuchs et al., 2013, 2014). Puntualmente, Fuchs et al. (2013) estudiaron el efecto moderador de la memoria de trabajo, el control atencional, la velocidad de procesamiento y la comprensión del lenguaje, en la relación entre diferentes tipos de instrucción matemática y el aprendizaje conceptual (sentido parte-entero, medición) y procedimental (cómputo aritmético) de las fracciones.

Por una parte, los resultados mostraron que la instrucción focalizada en la enseñanza del sentido de las fracciones como parte-entero y en los procedimientos de cómputo con fracciones generaba un mejor aprendizaje de la capacidad de comprender el sentido de medición de las mismas (estimación de línea numérica) y de computar operaciones aritméticas con fracciones en los niños con mayor capacidad de memoria de trabajo, comprensión del lenguaje y velocidad de procesamiento. Por otra parte, la instrucción focalizada en el sentido de medición de las fracciones producía un mejor aprendizaje del conocimiento conceptual de las fracciones como una relación parte-entero y como una forma de medición en los niños con mayor capacidad de memoria de trabajo y control atencional.

Asimismo, Fuchs et al. (2014) reportaron que la instrucción que se focalizaba principalmente en el sentido de las fracciones como medición generaba un mayor aprendizaje de la habilidad procedimental de estimación de la línea numérica con fracciones en los niños con menor capacidad de memoria de trabajo. En un sentido diferente, cuando este tipo de instrucción perseguía además la automatización de la capacidad de estimación en la línea numérica, la misma producía un mayor aprendizaje de tal capacidad procedimental en los niños con mayor capacidad de memoria de trabajo. 
En síntesis, los resultados de Fuchs et al. $(2013 ; 2014)$ indican que habilidades cognitivas de dominio amplio afectan el impacto del tipo de instrucción matemática recibida sobre el aprendizaje del conocimiento conceptual y procedimental.

\section{Evidencia a favor de perfiles individuales de desempeño}

En el último grupo de estudios analizados se encuentran las investigaciones que sugieren que existen diferencias inter-individuales en el grado de dominio respectivo de ambas formas de conocimiento de las fracciones, las cuales se reflejan en diferentes perfiles de conocimiento de estas.

El conjunto de perfiles identificados variaría en los diferentes estudios (ver Tabla 2), lo cual podría deberse a diferencias en las etapas de adquisición del conocimiento de las fracciones estudiadas (Hallet et al., 2012). Respecto de esta hipótesis, Hallet et al. (2012) mostraron que el número de perfiles se reducía de cuatro a dos en el transcurso de $6 .^{\circ}$ a $8 .^{\circ}$ año ${ }^{1}$. Asimismo, Hetch y Vagi (2012) reportaron que los perfiles presentarían escasa estabilidad en los primeros años de la adquisición del conocimiento de las fracciones (Hetch \& Vagi, 2012). Adicionalmente, las variaciones en el número de perfiles identificados podrían originarse en las distintas formas del conocimiento conceptual estudiadas. Por ejemplo, Hallet et al. (2010; 2012) utilizaron tareas que se centran en el sentido de las fracciones como medición y razón, mientras que Hetch y Vagi (2012) emplearon tareas que miden el conocimiento conceptual de adicción y el significado de las fracciones como una relación parte-entero y como una forma de medición de la magnitud.

Asimismo es importante destacar que la literatura sugiere que los perfiles de desempeño son independientes de la experiencia escolar, la inteligencia fluida y la capacidad de aprender procedimientos en general (Hallet et al., 2012) y que se asocian diferencialmente al desempeño general en fracciones (Hallet et al., 2010; Hetch \& Vagi, 2012). Concretamente, los niños que han exhibido un mayor conocimiento conceptual respecto del procedimental han mostrado un mejor desempeño general en el dominio de los números racionales y, dentro de estos, en el conocimiento general de las fracciones (Hallet et al., 2010; Hetch \& Vagi, 2012). 
Tabla 2. Perfiles del conocimiento identificados en estudios con análisis de clusters

\begin{tabular}{|c|c|c|c|c|c|}
\hline \multirow[b]{3}{*}{$\begin{array}{l}\text { Años de la } \\
\text { escolaridad } \\
\text { evaluados }\end{array}$} & \multicolumn{5}{|c|}{ Estudio } \\
\hline & Hallet et al. & \multicolumn{2}{|c|}{ Hallet et al. 2012} & \multicolumn{2}{|c|}{ Hetch \& Vagi (2012) } \\
\hline & $4 .^{\circ}$ y $5 .^{\circ}$ & $6 .^{\circ}$ & $8 .^{\circ}$ & $4 .^{\circ}$ & $5 .^{\circ}$ \\
\hline $\begin{array}{l}\text { Clusters } \\
\text { identificados* }\end{array}$ & $\begin{array}{l}\text { C1: c. } \\
\text { conceptual } \\
\text { bajo y c. } \\
\text { procedimental } \\
\text { más alto de lo } \\
\text { esperado } \\
\text { C2: c. } \\
\text { conceptual } \\
\text { bajo y c. } \\
\text { procedimental } \\
\text { alto } \\
\text { C3: c. } \\
\text { conceptual } \\
\text { próximo a } \\
\text { la media y c. } \\
\text { procedimental } \\
\text { bajo. } \\
\text { C4: c. } \\
\text { conceptual } \\
\text { alto y c. } \\
\text { procedimental } \\
\text { bajo. } \\
\text { C5: c. } \\
\text { conceptual y c. } \\
\text { procedimental } \\
\text { alto }\end{array}$ & $\begin{array}{l}\text { C1: c. } \\
\text { conceptual y c. } \\
\text { procedimental } \\
\text { bajos } \\
\text { C2: c. } \\
\text { conceptual } \\
\text { más alto de lo } \\
\text { esperado } \\
\text { C3: c. } \\
\text { procedimental } \\
\text { más alto de lo } \\
\text { esperado } \\
\text { C4: c. } \\
\text { conceptual y c. } \\
\text { procedimental } \\
\text { altos }\end{array}$ & $\begin{array}{l}\text { C1: c. } \\
\text { conceptual } \\
\text { más alto de lo } \\
\text { esperado } \\
\text { C2: c. } \\
\text { procedimental } \\
\text { más alto de lo } \\
\text { esperado }\end{array}$ & $\begin{array}{l}\text { C1: c. } \\
\text { conceptual } \\
\text { relativamente } \\
\text { más bajo de lo } \\
\text { esperado y c. } \\
\text { procedimental } \\
\text { esperado } \\
\text { C2: c. } \\
\text { conceptual } \\
\text { bajo y c. } \\
\text { procedimental } \\
\text { más alto de lo } \\
\text { esperado } \\
\text { C3: c. } \\
\text { conceptual } \\
\text { más alto de lo } \\
\text { esperado y c. } \\
\text { procedimental } \\
\text { relativamente } \\
\text { bajo } \\
\text { C4: c. } \\
\text { conceptual y } \\
\text { procedimental } \\
\text { relativamente } \\
\text { más altos de lo } \\
\text { esperado }\end{array}$ & $\begin{array}{l}\text { C1: c. } \\
\text { conceptual } \\
\text { bajo con } \\
\text { niveles } \\
\text { esperados } \\
\text { de c. } \\
\text { procedimental } \\
\text { C2: c. } \\
\text { conceptual } \\
\text { relativamente } \\
\text { bajo y c. } \\
\text { procedimental } \\
\text { alto } \\
\text { C3: c. } \\
\text { conceptual } \\
\text { esperado y c. } \\
\text { procedimental } \\
\text { más bajo de lo } \\
\text { esperado } \\
\text { C4: c. } \\
\text { conceptual } \\
\text { más alto de lo } \\
\text { esperado y c. } \\
\text { procedimental } \\
\text { relativamente } \\
\text { bajo } \\
\text { c5: c. } \\
\text { conceptual y } \\
\text { procedimental } \\
\text { relativamente } \\
\text { más altos de lo } \\
\text { esperado }\end{array}$ \\
\hline
\end{tabular}

* Nota: C: cluster; Puntuaciones altas o bajas conforme a lo esperado en función de las puntuaciones residuales correspondientes al otro tipo de conocimiento evaluado

\section{Discusión y conclusiones}

El objetivo de este trabajo fue analizar las relaciones que se establecen entre el conocimiento conceptual y el procedimental de las fracciones durante su aprendizaje. Para esto se efectuó una búsqueda bibliográfica cuyos resultados permitieron discriminar cuatro grupos de investigaciones: las que indican una relación bidireccional; las que sugieren una relación unidireccional; las que muestran cierta independencia o indican restricciones en la vinculación y, por último, aquellos trabajos que señalan diferencias inter-individuales en el grado de dominio respectivo de ambas formas de conocimiento.

Los resultados contradictorios entre los estudios podrían originarse en sus diferencias metodológicas. Por un lado, los distintos significados de las fracciones se asociarían diferencialmente a la capacidad de efectuar procedimientos con tal formato numérico (Charalambous \& Pitta-Pantazi, 2007; Fuchs et al., 2013) por lo cual, diferencias en el significado estudiado comprometerían las relaciones observadas. Por otro lado, los hallazgos disímiles respecto de la especificidad de la asociación entre ambos tipos de 
conocimiento podrían deberse a la etapa de adquisición y aprendizaje del conocimiento de las fracciones. Por ejemplo, Charalambous \& Pitta-Pantazi (2007) trabajaron con niños de $5 .^{\circ}$ y $6 .^{\circ}$ año cuya exposición a estímulos de aprendizaje de las fracciones se supone mayor a la de los participantes del estudio de Fuchs, los cuales cursaban $4 .^{\circ}$ año. Adicionalmente, el significado de las fracciones estudiado y el grado de aprendizaje de estas podrían explicar también las diferencias entre estudios en los perfiles de conocimiento identificados (Hallet et al., 2010; 2012).

Sumado a lo anterior, la forma en que el conocimiento conceptual y el procedimental de las fracciones se relacionan dependería del énfasis puesto en lo procedimental, lo conceptual o la interacción entre ambos durante su enseñanza (Mack, 1990; Osana \& Pitsolantis, 2011; Peled \& Segalis, 2005).

Desde otro ángulo, los resultados de Fuchs et al. (2013; 2014) indican que habilidades cognitivas de dominio amplio, como la memoria de trabajo, la atención o la velocidad de procesamiento moderan la asociación entre el conocimiento conceptual y el procedimental. Las investigaciones que analizaron la influencia del desarrollo cognitivo general sobre este tipo de relación son limitadas, por lo cual nuevos estudios serán necesarios para una comprensión más profunda de la influencia de terceras variables en la vinculación entre el conocimiento conceptual y el procedimental.

Es importante señalar que, independientemente del conocimiento procedimental, se observaron diferencias y algunas dificultades en la medición del conocimiento conceptual que podrían afectar la dirección de las asociaciones halladas en diferentes estudios (problema de la impureza) (Schneider \& Stern, 2010). Schneider y Stern (2010) reportaron que las tareas típicamente utilizadas para evaluar ambos aspectos del conocimiento presentan notorios problemas de validez, los que se originan en que estas presentan fuentes de varianza ajenas al dominio del conocimiento evaluado (e.g., varianza asociada al nivel de vocabulario). Asimismo, los niños serían capaces de efectuar ciertos procedimientos en función de su conocimiento conceptual y de comprender ciertos conceptos en base a su experticia en la ejecución de procedimientos, por lo cual resulta complejo controlar la influencia recíproca entre ambas formas de conocimiento al momento de su medición.

El grado en que una tarea puede ser considerada un modo de medición de una habilidad procedimental depende del grado de entrenamiento y práctica para la realización de la misma. Por ejemplo, la estimación de fracciones en la línea numérica es considerada por numerosos autores una tarea que evalúa el conocimiento conceptual (Charalambous \& Pitta-Pantazi, 2007; Fuchs et al., 2013; 2014; Hallet et al., 2010). Sin embargo, cuando se utiliza durante la enseñanza y el niño incorpora secuencias de pasos para su realización (e.g., segmentar la línea en "x" partes iguales, ubicar el número conforme a su carácter mayor o menor respecto de "x" magnitud de referencia) se evaluaría un conocimiento procedimental (Rittle et al., 2001). De este modo, la clasificación de una tarea como una forma de evaluación de conocimientos conceptuales o procedimentales depende en parte del tipo de enseñanza que el alumno recibe, así como del grado de aprendizaje y recuperación automatizada de los conocimientos.

En síntesis, los trabajos revisados aportan evidencias inconsistentes que no permiten establecer una conclusión firme sobre el sentido de la relación entre el conocimiento conceptual y el procedimental de las fracciones. Para esclarecer la relación entre ambos, futuras investigaciones deberían explorar relaciones específicas entre los diferentes significados conceptuales de las fracciones y los procedimientos. Asimismo, deberían controlar el efecto del tipo de enseñanza (e.g., instrucción centrada en los conceptos, 
los procedimientos, ambos o su interacción) sobre el desarrollo de ambas formas de conocimiento y su relación. Adicionalmente, sería conveniente controlar las diferencias individuales en habilidades cognitivas de carácter general tales como la memoria de trabajo, el control atencional, la velocidad de procesamiento y la comprensión del lenguaje, entre otras.

Por último, sería necesario medir ambos aspectos del conocimiento matemático controlando la impureza de las tareas.

Se espera que este trabajo represente una contribución para futuras investigaciones en el área al señalar la importancia de cuidar y controlar diferentes aspectos que ejercen influencia en el establecimiento de relaciones en el aprendizaje del conocimiento conceptual y el procedimental de las fracciones. La comprensión adecuada de estas relaciones contribuirá al enriquecimiento de los modelos teóricos del desarrollo de la cognición matemática, los que resultan esenciales para diseñar estrategias efectivas de enseñanza de contenidos matemáticos así como técnicas para la identificación y tratamiento de niños con dificultades en su aprendizaje.

\section{Referencias bibliográficas}

Ancker, J. S. \& Kaufman, D. (2007). Rethinking health numeracy: a multidisciplinary literature review. Journal of the American Medical Informatics Association, 14(6), 713-21.

Baron, R. M. \& Kenny, D. A. (1986). The moderator-mediator variable distinction in social psychological research: Conceptual, strategic, and statistical considerations. Journal of personality and social psychology, 51(6), 1173.

Baroody, A. J. (2003). The development of adaptive expertise and flexibility: the integration of conceptual and procedural knowledge. Mahwah, Nueva Jersey: Lawrence Erlbaum Associates.

Behr, M.J.; Harel, G.; Post, T. \& Lesh, R. (1993). Rational numbers: Toward a semantic analysis-emphasis on the operator construct, en T.P. Carpenter, E. Fennema and T.A. Romberg (eds.), Rational Numbers: An Integration of Research pp. 13-47. Nueva Jersey: Lawrence Erlbaum Associates.

Bisanz, J. \& LeFevre, J. A. (1992). Understanding elementary mathematics. Advances in psychology, 91, 113-136.

Byrnes, J. P. \& Wasik, B. A. (1991). Role of conceptual knowledge in mathematical procedural learning. Developmental Psychology, 27(5), 777.

Charalambous, C. Y. \& Pitta-Pantazi, D. (2007). Drawing on a theoretical model to study students' understandings of fractions. Educational Studies in Mathematics, 64(3), 293-316.

Clark, K. E. (1988). The importance of developing leadership potential of youth with talent in mathematics and science. En J. Dreyden; S. A. Gallagher; G. E. Stanley \& R. N. Sawyer (Eds.), Report to the National Science Foundation: Talent Identification Program/National Science Foundation Conference on Academic Talent (pp. 95-104). Durham, North Carolina: National Science Foundation.

Fuchs, L. S.; Schumacher, R. F.; Long, J.; Namkung, J.; Hamlett, C. L.; Cirino, P. T. ... \& Changas, P. (2013). Improving at-risk learners' understanding of fractions. Journal of Educational Psychology, 105(3), 683. 
Fuchs, L. S.; Schumacher, R. F.; Sterba, S. K.; Long, J.; Namkung, J.; Malone, A.; ... \& Changas, P. (2014). Does working memory moderate the effects of fraction intervention? An aptitudetreatment interaction. Journal of Educational Psychology, 106(2), 499.

Fuson, K. C. (1998). Pedagogical, mathematical, and real-world conceptual-support nets: A model for building children's multidigit domain knowledge. Mathematical Cognition, 4(2), 147-186.

Geary, D. C. (1994). Children's mathematical development: Research and practical applications. American Psychological Association.

Gelman, R. \& Williams, E. M. (1998). Enabling constraints for cognitive development and learning: Domain specificity and epigenesis.

Greeno, J. G.; Riley, M. S. \& Gelman, R. (1984). Conceptual competence and children's counting. Cognitive Psychology, 16(1), 94-143.

Grinyer, J. (2005). Literacy, numeracy and the labour market. Londres: DfES

Halford, G. S. (2014). Children's understanding: The development of mental models. Psychology Press.

Hallett, D.; Nunes, T. \& Bryant, P. (2010). Individual differences in conceptual and procedural knowledge when learning fractions. Journal of Educational Psychology, 102(2), 395.

Hallett, D.; Nunes, T.; Bryant, P. \& Thorpe, C. M. (2012). Individual differences in conceptual and procedural fraction understanding: The role of abilities and school experience. Journal of Experimental Child Psychology, 113(4), 469-486.

Hecht, S. A. \& Vagi, K. J. (2010). Sources of group and individual differences in emerging fraction skills. Journal of educational psychology, 102(4), 843.

Hecht, S. A. \& Vagi, K. J. (2012). Patterns of strengths and weaknesses in children's knowledge about fractions. Journal of Experimental Child Psychology, 111(2), 212-229.

Hiebert, J., \& Wearne, D. (1986). Procedures over concepts: The acquisition of decimal number knowledge.

Hiebert, J. (2013). Conceptual and procedural knowledge: The case of mathematics. Routledge.

Hoffer, T. B.; Venkataraman, L.; Hedberg, E. C. \& Shagle, S. (2007). Final report on the national survey of algebra teachers for the National Math Panel. Retrieved March, 25, 2011.

Karmiloff-Smith, A. (1992). Beyond Modularity: A Developmental Perspective on Cognitive Science. Cambridge, MA: MIT Press.

Kerslake, D. (1986). Fractions: Children's Strategies and Errors. A Report of the Strategies and Errors in Secondary Mathematics Project. Berkshire: NFER-NELSON Publishing Company, Ltd. 
Kieren, T. E. (1976). On the mathematical, cognitive, and instructional foundations of rational numbers, en R. Lesh (ed.), Number and Measurement: Papers from a Research Workshop ERICI SMEAC, pp. 101-144. Columbus.

Kieren, T. E. (1993). Rational and fractional numbers: From quotient fields to recursive understanding. Rational numbers: An integration of research, 49-84.

Mack, N. K. (1990). Learning fractions with understanding: Building on informal knowledge. Journal for research in mathematics education, 16-32.

National Mathematics Advisory Panel (2008). Foundations for success: The final report of the national mathematics advisory panel. Washington, DC: U.S. Department of Education. Recuperado de http://www2.ed.gov/about/bdscomm/list/mathpanel/report/final-report. pdf

Osana, H. P. \& Pitsolantis, N. (2013). Addressing the struggle to link form and understanding in fractions instruction. British Journal of Educational Psychology, 83(1), 29-56.

Paglin, M. \& Rufolo, A. M. (1990). Heterogeneous human capital, occupational choice, and male-female earnings differences. Journal of Labor Economics, 8, 123-144.

Parsons, S. \& Bynner, J. (2005). Does numeracy matter more? National Research and Development Centre for Adult Literacy and Numeracy. Research Report London: Institute of Education. Recuperado de: http://eprints.ioe.ac.uk/4758/1/parsons2006does.pdf

Peck, D. M. \& Jencks, S. M. (1981). Conceptual issues in the teaching and learning of fractions. Journal for Research in Mathematics Education, 339-348.

Peled, I. \& Segalis, B. (2005). It's not too late to conceptualize: Constructing a generalized subtraction schema by abstracting and connecting procedures. Mathematical Thinking and Learning, 7(3), 207-230.

Rittle-Johnson, B. \& Schneider, M. (2014). Developing conceptual and procedural knowledge of mathematics. En R. Kadosh \& A. Dowker (Eds), Oxford Handbook of Numerical Cognition. Oxford University Press.

Rittle-Johnson, B.; Schneider, M. \& Star, J. R. (2015). Not a One-Way Street: Bidirectional Relations between Procedural and Conceptual Knowledge of Mathematics. Educational Psychology Review, 1-11.

Rittle-Johnson, B. \& Siegler, R. S. (1998). The relation between conceptual and procedural knowledge in learning mathematics: a review. En C. Donlan (Ed.), The Development of Mathematical Skills (pp. 75-110). Londres: Psychology Press.

Rittle-Johnson, B.; Siegler, R. S. \& Alibali, M. W. (2001). Developing conceptual understanding and procedural skill in mathematics: an iterative process. Journal of Educational Psychology, 93, 346-362. doi: 10.1037//0022-0663.93.2.346 
Rivera-Batiz, F.L. (1992). Quantitative literacy and the likelihood of employment among young adults in the United States. Journal of Human Resources, 27 (2), 313-328.

Rose, H. \& Betts, J.R. (2004). The effect of high school courses on earnings. Review of Economics and Statistics, 86 (2), 497-513.

Sadler, P. M., \& Tai, R. H. (2001). Success in introductory college physics: The role of high school preparation. Science Education, 85(2), 111-136.

Schneider, M. \& Stern, E. (2010). The developmental relations between conceptual and procedural knowledge: A multimethod approach. Developmental Psychology, 46(1), 178.

Siegler, R. S.; Duncan, G. J.; Davis-Kean, P. E.; Duckworth, K.; Claessens, A.; Engel, M.; ... \& Chen, M. (2012). Early predictors of high school mathematics achievement. Psychological science, 23(7), 691-697.

Siegler, R. S. \& Stern, E. (1998). Conscious and unconscious strategy discoveries: a microgenetic analysis. Journal of Experimental Psychology: General, 127, 377-397. doi: 10.1037/0096-3445.127.4.377.

Siegler, R. S.; Thompson, C. A. \& Schneider, M. (2011). An integrated theory of whole number and fractions development. Cognitive psychology, 62(4), 273-296.

Vamvakossi, X. \& Vosniadou, S. (2004). Understanding the structure of the set of rational numbers: A conceptual change approach. Learning and Instruction, 14(5), 453-467.

Vamvakossi, X. \& Vosniadou, S. (2010). How many decimals are there between two fractions? Aspects of secondary school students' understanding of rational numbers and their notation. Cognition and instruction, 28(2), 181-209.

Vizcarra, R. E. \& Sallán, J. M. G. (2005). Modelos de medida para la enseñanza del número racional en Educación Primaria. Unión: Revista Iberoamericana de Educación Matemática, (1), 17-35.

Yoshida, H. \& Sawano, K. (2002). Overcoming cognitive obstacles in learning fractions: Equal "partitioning and equal"whole. Japanese Psychological Research, 44(4), 183-195.

\section{(Endnotes)}

${ }^{1}$ Los alumnos de $6 .^{\circ}$ año pertenecen al nivel educativo primario del sistema educativo de Inglaterra, mientras que los de $8 .^{\circ}$ año pertenecen al nivel secundario. En el primer caso presentan edades entre 10 y 11 años y en el segundo edades entre 12 y 13 años. 